\title{
Research of Product Classifying and Coding Based on the Things Characteristics and Realization of the Relevant Management System
}

\author{
Chen Xi ${ }^{\text {a }}$, Hao Lijian ${ }^{\text {a }}$, Yang Guichun ${ }^{\text {a }}$, Zhang Jiali ${ }^{\mathrm{a}}$, Xia Guijian ${ }^{\mathrm{a}}$, Ma Yanli ${ }^{\mathrm{a}}$ \\ ${ }^{a}$ China North Engine Research Institute, Datong 037036, China
}

\begin{abstract}
In this paper, the advantages and disadvantages of three kinds of information code (subjection code, composite code and parallel code) were analyzed and compared. It was put forward that adopt parallel code method based on things characteristics realizing the information classifying and coding management of the whole life cycle of the products. And the structure model of product classifying code based on parallel code method was established. At the same time, the implementation method of product classifying code was presented for application requirements of the enterprise. Finally, the classifying and coding management system of product was introduced that supported by ORACLE and used JSP to development.
\end{abstract}

Index Terms: classifying code; identification code; attribute cell; multiple classification; code management system

(c) 2012 Published by MECS Publisher. Selection and/or peer review under responsibility of the Research Association of Modern Education and Computer Science.

\section{Introduction}

Enterprise informationization is an important part of the national economy informationization; enterprise informationization is the important means that enterprises realize by the extensive management to intensive management; enterprise informationization is the important ways to improve enterprise modern management level and enhance enterprise competitiveness. However, in the process of informatization construction, due to emphasizing technology and system unilaterally, many domestic enterprises have ignored the basic informationization work of classifying and coding uniformly for information. Accordingly the phenomenon of "information island" appear. Therefore, it is the fundamental guarantee and the basic work of implementing informatization successfully that enterprises establish uniform, simple, perfect, no alternative classifying code for product information. Finally, by code all information of product will be searched quickly and used efficiently; fast development and design of product will be achieved; market competitiveness of enterprise will be enhanced largely, too.

* Corresponding author.

E-mail address: chx_chenxi@126.com 


\section{Coding Method Research}

\subsection{Coding Method}

Now there are three kinds of the common coding methods including subjection code, composite code and parallel code [1]. The subjection code method is a method used widely. By this code users can gain the using information of parts easily (i.e. assembly location and relationship). So, the code method is helpful for guiding product assembly process; the code method applies to special machinery products industries that have various product sorts, one-piece or small batch production and the phenomenon of borrowing parts little, such as engineering machinery industry. Therefore, this code method adapts to these enterprises of which information management just started and production planning was weaker and production only depended on experience. But the subjection code cannot obtain basic information of parts, which is unfavorable to organize production, which is unfavorable to manage and reuse information. Therefore this code method is easy to cause confusion of management. The composite code consists of classifying code and identification code. Two parts are associated with fixed form. The identification code attaches to the classifying code. This code is usually only suitable for special occasions, of which main weakness is difficult to extend. The parallel code includes classifying code based on things characteristics and identification code. At the same time, classifying code and identification code of parallel code is independent of each other. The advantage of this code is that users can understand the basic information of parts by things characteristics. So this code method is helpful for reusing parts and organizing production; and it is especially suitable for batch products, of which characteristics are quite strong production planning, batch production, less product categories, universal property and borrowing parts frequently. Classifying code embodies the classification information of coding object, which is facilitate users to effectively retrieval and reusing of design data. Identification code ensures the uniqueness of coding object. Combination code of classifying code and identification code is convenient to computer processing; and it is effective to save storage space and reduce error ration of code; and it has good scalability. So this form of coding system has been used widely.

Thus, with an enterprise application as the starting point, this paper adopts parallel encoding method realizing the information classifying and coding management of the whole life cycle of the products.

\subsection{Coding Structure Model}

According to the research result of coding method and actual need of enterprise information coding, the paper put forward that code structure is composed of classifying code, identification code and characteristic attribute. At the same time, users can define classifying code and identification code dynamically according to different objects.

Main function of classifying code is to categorize on the basis of object properties. In the German industrial standard DIN4000 and Chinese GB 10091 there have been guidance suggestions for establishing of classifying code [2]. In practical applications, according to specific application needs, different classification system can be set up, such as design classification, material classification, standard parts classification, procurement classification, etc. Different classification systems have different classifying code[3]; multiple index relations can be set up between classifying codes. Identification codes are the number that is used to distinguish and differentiate different objects; because identification codes must be uniqueness, usually identification codes are sequence numbers generated automatically by the computer. To those enterprise, which parts code system have been set up , as long as these parts codes are uniqueness, these codes also can be used as identification codes. Thus, because old code will not be replaced, in the process of implementing code the pressure from all sides will be reduced. Attribute cell is used to describe the common things feature of objects (i.e. the key attributes of objects). Through attribute pool attribute cell is managed uniformly, which will ensure the uniqueness of all attribute names and facilitate to control and standardize and query for 
attributes. In the process of product development and design, users can gain relevant attributes from the definition list of attribute pool according to the different needs. But attributes in attribute pool cannot be added optionally. If the parameter must be added, users apply for requires and the administrator who have certain privileges of system management add the parameter in attribute pool.

The system creates code dynamically from left to right, according to different code segment. Finally, both classifying code and identification code make up whole code. The product coding structure model based on parallel code method is shown in fig. 1.

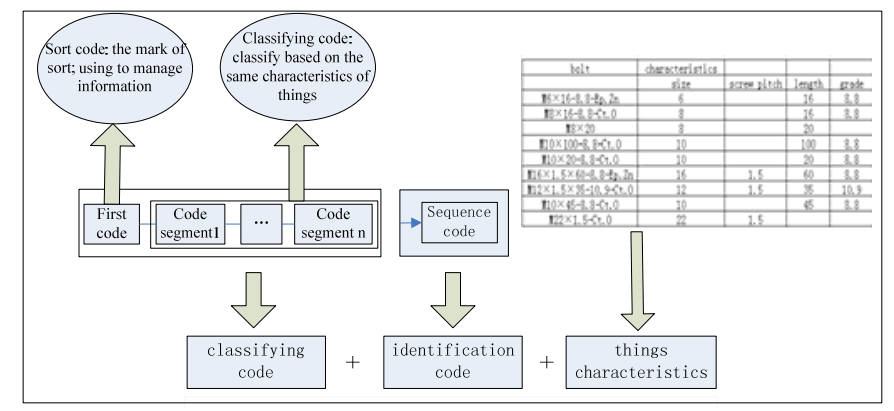

Fig.1. Product Coding Structure Model

\section{Implementation of Classifying code}

Classifying and coding for Product information is a systemic project involving the informatization construction of entire enterprise. Therefore implementation of coding must be based on requires of enterprise and be easy to use as a starting point. However, in the current theory, only basic principles and basic methods of information classifying and coding can be got and specific process of implementation can not be gained.

On the basis of the existing theories, the workflow of product classifying and coding base on parallel code method was constructed, which included the preparation stage, the creating stage and the application stage, as shown in the fig. 2.

\subsection{Preparation Stage}

In the preparation stage the main task is to analyze objects of enterprise coding in the round and in detail, and to complete analyzing report. The content of the report include analysis for objects' generality, analysis for objects' names and analysis for objects' characteristics. Because the range of these foundational works involve very wide, consisting of the information department, the standardization department, the design department, the manufacture department and sale department, these works need support of enterprise's highlevel people and depend on collaboration among the various departments.

1) Analysis of Objects’ Generality

Ensuring method of Objects' generality includes the qualitative ABC analysis method and quantitative spectrum statistics method. Combining the actual conditions of implementing enterprise, ABC analysis method was adopted in this paper. The goal of ABC analysis as follows: objects will be changed as far as possible from class B to class C, i.e. typical self-made parts and related resources of enterprise will be changed into standard or outsourcing parts; objects will be changed as far as possible from class A to class B, i.e. special parts and related resources of enterprise will be changed into typical self-made parts. Through ABC analysis of objects, enterprises can find out the target of implementing standardization, and reduce designing and manufacturing depth, and finally realize to focus on core technology. 


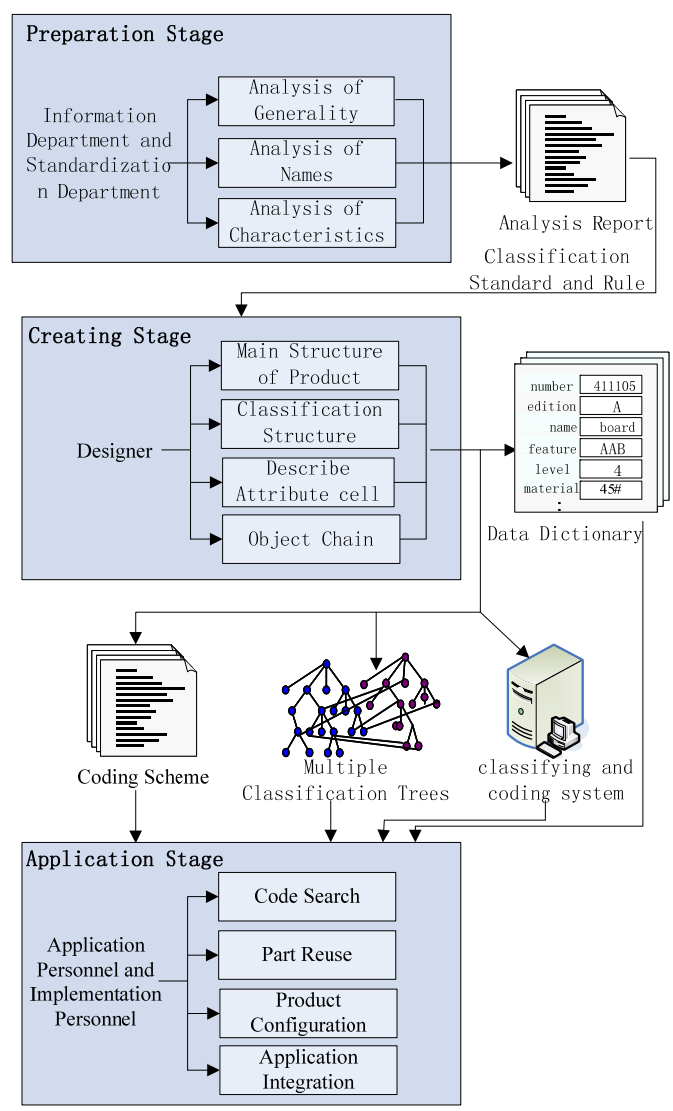

Fig.2. Work Flow of Construct Product Classifying Code

\section{2) Analysis of Objects’ Names}

The main purpose of objects' names analysis is to establish a unified and non-redundant name dictionary. When the developer and designers need name a new object, they can only change appropriate name from this name dictionary. Data management system refuses to accept the name of the object which is inexistent in the data dictionary. If it is necessary to add a new name of object in the data dictionary, a strict process of standardization must be implemented. After regulating the name of object, the phenomenon of parts naming chaos will be reduced greatly and the reusability of parts will be enhanced tremendously.

3) Analysis of Objects' Characteristics

Products and parts of enterprises are various, but those products and parts have some common features. Through some observation and analytical methods (such as geometrical/topological feature analysis), the same features can be abstracted to form things characteristics. Then all thing characteristics are collected to form a book. The book will be helpful to improve the efficiency of work. 


\subsection{Creating Stage}

According to analysis report, combining coding rule, classification standard and regulation, designer establish main structure of product, classification structure of object and object chain, collect and describe characteristic attribute of object, and constructs the coding scheme, the data dictionary, multiple classification trees, classifying and coding system.

1) Establishing Main Structure of Product

For the product structure of implementing enterprise, a configurable and modularized product system will be established, which include all standard parts and non-standard parts. According to different needs of users, product structure customized by users can be derived from main structure of product.

2) Establishing Classification Structure of Object

Before the designer establishes the data dictionary, classification structure of parts resources based on part class will be built firstly. The reasonable classification structure not only facilitates users to query information of parts, but also directly affects expanded capacity of parts database. When establishing classification structure of parts resources, the basic rules as follows: range covering rules and maximum applicability rules.

3) Collect and Describe Attribute Cell

The list of attribute cell is the information collection having the same characteristics. Attribute cell describes the same features of similar objects.

4) Forming Object Chain

The objects chain of product cover all the associative products information of the whole life cycle, which include all engineering drawings, documentation, model, product structure, thing characteristics connection with the product.

5) Compiling Data Dictionary

After building classification structure and described rules of attribute cell, the designers will establish the data dictionary for describing objects. The data dictionary stores the term, rules and mutual relationship standardized and regulated, which is the foundation establishing classification model of product information in enterprise. The function of the data dictionary is to regulate all parameters used in product designing and manufacturing work. When designers are working, they can select parameters needed from the data dictionary. But they cannot add parameters randomly. If some parameters must be added, designers apply for requires and the administrator add parameters into the data dictionary. After constructing the data dictionary, the content and express standard of the data dictionary must be checked by high level experts, which will ensure the correctness and normalization of information.

6) Constructing Multiple Classification Trees

According to user needs, classification tree is established by selecting most important characteristics of object, for example three classification trees can be built according to the different feature of standard parts, special parts and borrowed parts. The classification tree expresses a kind of inheriting relationship. Each kind has own attributes, simultaneously an inferior kind inherits attributes of superior kind. Thus the collection of all kinds combines into the multiple classification trees. Main structure of product is a structure tree established by the membership relation of product assembly, which not only consists of all parts of product, but also includes related version information. Main structure of product and multiple classification trees will be related together. Every node in classification tree is a class, which is marked by classification code. Each node in main structure of product is corresponding to every instance of class in classification tree. Every instance in classification tree is marked by identification code. Through this relationship, user can find and obtain the class information of object conveniently from main structure of product, and get classification code of object from classification tree. By comparing a class of object and analyzing the using and designing case of this class parts, thus the goal will be achieved to decrease the repeated design of parts. Combining with the thinking mode of designer, according to different requirements, enterprises can establish various multiple classification trees to satisfy searching needs of user. 


\subsection{Application Stage}

Firstly, coding scheme ensured is instantiated. Secondly, classifying and coding system is developed and established。Coding system realizes the uniform management of product information involving each stage of life cycle. Through the coding system, designers will be easy to quickly find and reuse for all information. Finally, according to the actual needs of enterprises, for integration of coding system and the other application system (such as product data management system, process management system and material management system) some interfaces need be developed to realize two-way exchange of data.

\section{Designing and Realizing of the Classifying and Coding Management System}

\subsection{System Designing}

In the paper, the classifying and coding management system is improved on the basis of general coding management mode. On the one hand, this system realizes some basic functions [4], such as customizing the classification and coding rules of enterprise, automatically giving code, finding code and so on. On the other hand this system has some else functions, such as building multiple classification trees, management of characteristic attribute, user management and log management. Through these function, this system can satisfy the using request of users. The whole function frame of coding system is shown in fig. 3 .

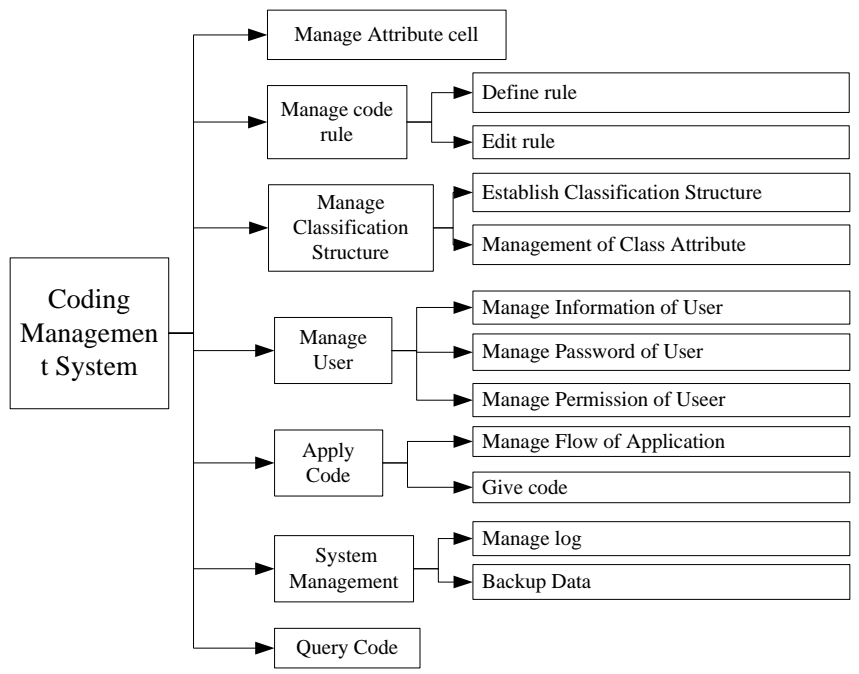

Fig.3. Function Frame of Coding System

\subsection{System Realizing}

The classification and coding management system adopts B/S structure, and the development language of system is JSP, and database is Oracle, main interface of system shown as shown in fig. 4. At present the system has been successfully applied in china north engine research institute. The system runs in good condition and achieves the desired effect to use 


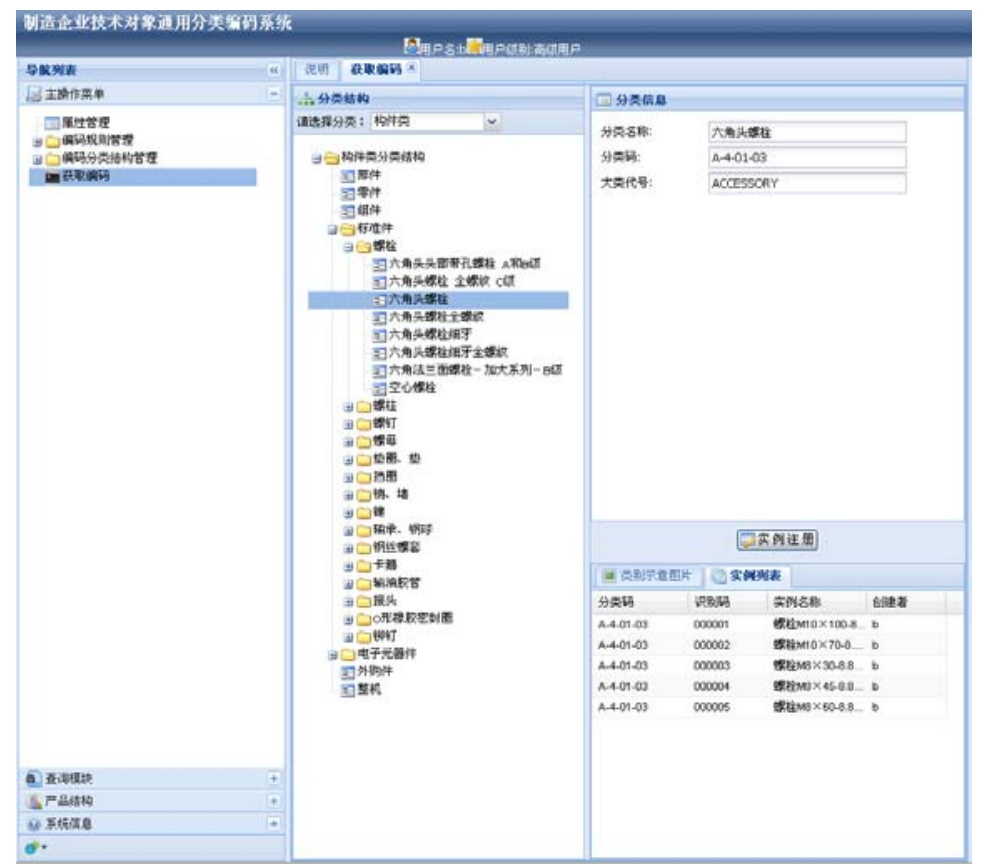

Fig.4. Interface of Classifying and Coding Management System

\section{Conclusion}

In this paper, through parallel code method based on things characteristics realized the information classifying and coding management of the whole life cycle of the products. And the classifying and coding management system of product on the basic of classifying code was developed and built. Finally, the system was successfully applied in china north engine research institute. The speed of searching information in this enterprise was increased largely by the system.

\section{References}

[1] [T Warren Liao, 2001] T. Warren Liao. Classification and coding approaches to part family formation under a fuzzy environment [J]. Fuzzy Sets and Systems, 2001, 122: 425-441.

[2] LI Ying-hua. On Problem Classification and Coding for Two-dimensional Geometric Nesting[J]. MECHANICAL SCIENCE AND TECHNOLOGY, 2000.

[3] Xie Xi-hua, He Qing-hua,Zhou Liang. Classification coding mode of PDM based on flexible structure [J]. Computer Integrated Manufacturing Systems, 2005.

[4] [Joerg, 2002] Joerg Leukel, Volker Schmitz, Frank-Dieter Dorloff. A Modeling Approach for Product Classification Systems[C]. Proceedings of the 13th International Workshop on Database and Expert Systems Applications (DEXA'02), 2002. 